\title{
NEAR-IR SPECTROSCOPY OF THE ATMOSPHERE OF JUPITER
}

\author{
R.W. CARLSON AND K.H. BAINES \\ Jet Propulsion Laboratory \\ California Institute of Technology \\ Pasadena, CA 91109 USA \\ T. ENCRENAZ, P. DROSSART AND M. ROOS-SEROTE \\ Observatoire de Paris-Meudon \\ Departement Spatiale \\ F-92195 Meudon, France \\ AND \\ F.W. TAYLOR, P. IRWIN, A. WEIR, P. SMITH AND S. CALCUTT \\ Oxford University \\ Clarendon Laboratory \\ Oxford, OX1 3PU, UK
}

\begin{abstract}
The Galileo Near Infrared Mapping Spectrometer (NIMS) obtains spectral images in the wavelength range 0.7 to $5.2 \mu \mathrm{m}$ with a spectral resolving power of approximately 200 . This spectral range allows NIMS to sense cloud-reflected solar radiation, thermal emission produced in the deep atmosphere, and auroral emission from the thermosphere of Jupiter. Using $5 \mu \mathrm{m}$ thermal emission spectroscopy, the amount of water vapor in the deep atmosphere, at approximately the 6 to 8 bar level, is found to vary by a factor $>100$. Deep atmosphere ammonia was also found to vary, with a spatial behavior different from that of water vapor. No evidence is found for a massive water cloud. Using reflected solar radiation in conjunction with thermal emission, two cloud layers are found, the upper at 0.5 bars and a lower one at 1-1.3 bars. The inferred absorption properties of these clouds are consistent with ammonia crystals (the upper cloud) and ammonium hydrosulfide particles (the lower cloud).
\end{abstract}

\section{Introduction}

Combining spectroscopic and imaging capabilities - imaging spectroscopy -- provides a powerful method for planetary studies. Diverse physical processes in the Jupiter atmosphere can be investigated through infrared spectra, ranging from high altitude aurora, reflected solar radiation from the troposphere, and thermal emission from the deep atmosphere. With mapping capabilities, the organization and spatial variations of atmospheric features can be elucidated. The Near Infrared Mapping Spectrometer experiment (NIMS) on the Galileo spacecraft is being used to study the compositional variations in the atmosphere, the structure of the clouds and cloud particle microphysics, and auroral phenomena. The NIMS instrument (Carlson et al. 1992) obtains spectra over the wavelength range 0.7 to $5.2 \mu \mathrm{m}$ at modest spectral resolution $(0.025 \mu \mathrm{m}$ for wavelengths above $1 \mu \mathrm{m}, 0.013 \mu \mathrm{m}$ below). The angular resolution of $0.5 \mathrm{mrad}$ results in a spatial pixel size of typically 350 to $500 \mathrm{~km}$ on Jupiter's atmosphere. Highlights of some of these results are presented below.

\section{Global Observations}

An example of the global measurements is illustrated in Fig. 1 (see color plates), which shows the face of Jupiter containing the Great Red Spot. The planet was observed at five wavelengths, including two "continuum" wavelengths $(1.61$ and $2.73 \mu \mathrm{m})$, a wavelength $(2.17 \mu \mathrm{m})$ which is strongly 

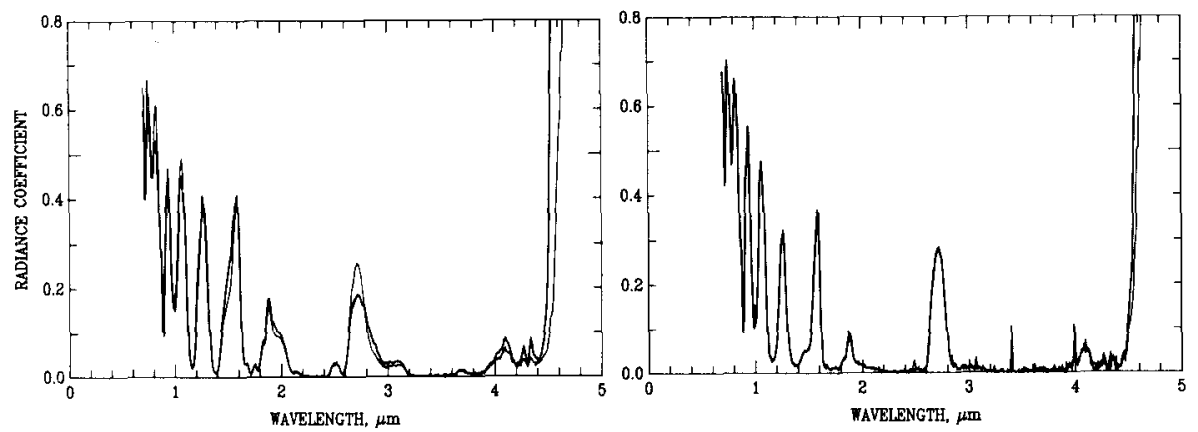

Figure 2. Reflectance spectra of Jupiter. The two spectra in the upper panel are from a hot spot region near $6^{\circ} \mathrm{N}$. latitude with an incidence angle of $42^{\circ}$, an emission angle of $13^{\circ}$ and a phase angle of $46^{\circ}$. Corresponding parameters for the spectra in the lower panel are: $60^{\circ} \mathrm{N}, 66^{\circ}, 62^{\circ}$, and $46^{\circ}$, respectively. The onset of the thermal emission spectrum appears at the edge of a phosphine absorption band at approximately $4.2 \mu \mathrm{m}$. Each pair of spectra show differing amounts of thermal emission. Corresponding reflectance changes are apparent in the upper spectra, which have lower air mass. Reflectance is expressed as the radiance coefficient, which is the ratio of the observed brightness to that of a Lambertian surface, illuminated at the same angle. The spectra are from the first and third orbits (G1 and $\mathrm{C} 3$ )

affected by molecular hydrogen pressure-induced absorption and probes the high stratosphere and lower stratosphere, a wavelength strongly absorbed by ammonia gas $(3.01 \mu \mathrm{m})$, and a wavelength $(4.99 \mu \mathrm{m})$ which senses thermal radiation emanating from the deep troposphere and is an indicator of cloud transparency.

The Great Red Spot is seen to reach relatively high altitudes as shown in the $2.17-\mu \mathrm{m}$ image, which also shows polar hazes and a high altitude haze over the southern boundary of the North Equatorial Belt. Reflected radiation at $3.01 \mu \mathrm{m}$ also senses these high hazes as well as the upper cloud layer, long thought to be composed of ammonia crystals. This image is bland compared to those of the continuum channels, indicating that the cloud layers (the postulated ammonium hydrosulfide clouds) probed by these more deeply penetrating channels exhibit greater spatial variability than the $\mathrm{NH}_{3}$ cloud above. Indeed, it is thinning of these lower clouds that produces the spatially variable thermal emission pattern seen in the $4.99-\mu \mathrm{m}$ image.

\section{Reflected Sunlight Spectra and Cloud Properties}

NIMS obtains both reflectance and thermal emission spectra, and this combination allows simultaneous study and retrieval of cloud properties and composition. Representative spectra for the reflected-sunlight spectral region are shown in Fig. 2, which also shows the rise in thermal emission longward of $4 \mu \mathrm{m}$. The left two spectra are from an equatorial latitude while the two on the right are from $60^{\circ} \mathrm{N}$ latitude. In both locations, there were spatial variations in the thermal radiation from one pixel to the next, so the two spectra at each location are different.

Gaseous absorption bands determine the shape of the spectrum. Methane is a major absorber and ammonia absorption is also evident in the $0.7-1.1,1.5,1.95$, and $3 \mu \mathrm{m}$ regions, where the equatorial spectra show variations. Pressure induced absorption by $\mathrm{H}_{2}$ occurs between 2.0 and 2.2 $\mu \mathrm{m}$, and phosphine absorption is found at $4.2 \mu \mathrm{m}$ and above.

Since methane and hydrogen are well-mixed and stable gases, their absorption bands can be used to determine the cloud structure and ammonia variations. A forward retrieval method, developed at Oxford University, uses the full $0.7-$ to $5.2-\mu \mathrm{m}$ spectrum to determine the cloud pressure levels, optical opacities, particle sizes and local ammonia and water vapor abundances. The analysis scheme uses full scattering solution and the correlated-k technique for gaseous absorption (Irwin et al. 1996). Particulate scattering and absorption is computed from Mie scattering theory and the optical constants for ammonia and ammonium hydrosulfide. Results for the cloud parameters (Irwin et al. 1997), using the equatorial spectra of Fig. 2, indicate the occurrence of an upper haze at a pressure level of $150 \mathrm{mb}$, consisting of $1 \mu \mathrm{m}$ diameter tholin particles. A cloud consistent with ammonia crystals occurs at $600 \mathrm{mb}$ with particle diameters of approximately $1.5 \mu \mathrm{m}$. At lower altitudes, a cloud whose optical properties are consistent with $\mathrm{NH}_{4} \mathrm{SH}$ is found at 1.3 bars and consists of $\sim 0.9$ 


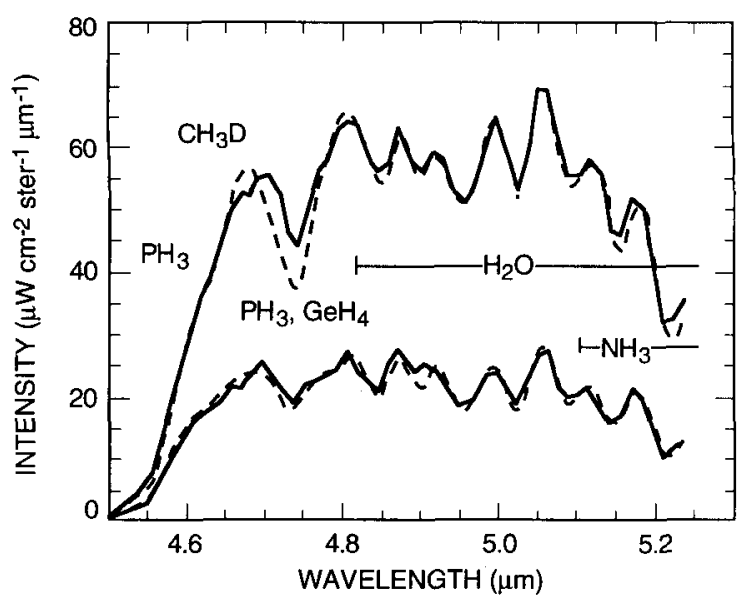

Figure 3. Two spectra from a thermal emission spectral mapping sequence (a HOTMAP sequence from the fourth orbit). The approximate regions of molecular absorption are indicated. The solid lines are NIMS observations, and correspond to the locations indicated by $x$ 's in Fig. 4 (the lower spectrum corresponds to the left location). The dashed lines are model fits with the following parameters for the upper (lower) spectra: water relative humidity, $0.2 \%$ $(5 \%)$; cloud transmission, $0.50,(0.32)$; relative ammonia concentration, $0.5,(0.75)$.

$\mu \mathrm{m}$ diameter particles. A variable component of this cloud, or a somewhat lower cloud, with larger particle sizes ( $>2 \mu \mathrm{m}$ diameter) is responsible for the spatially variable cloud extinction of $5 \mu \mathrm{m}$ thermal radiation. No evidence for a deep water cloud was found in this analysis.

\section{Thermal Emission Spectra and Water Abundance}

A spectral window occurs in the $5-\mu \mathrm{m}$ region, which allows thermal radiation produced in the lower troposphere to escape the atmosphere, providing that the overlying cloud cover is not opaque. The primary mechanisms which produce this thermal emission are collision-induced rotation-translation transitions of $\mathrm{H}_{2}$ and radiation from the proposed water clouds. This thermal radiation can be absorbed by molecules above the emíssion region, specifically $\mathrm{H}_{2} \mathrm{O}, \mathrm{NH}_{3}, \mathrm{CH}_{3} \mathrm{D}, \mathrm{PH}_{3}$, and $\mathrm{GeH}_{4}$. The latter two molecules are produced much deeper in the atmosphere, at the 1000 to $2000 \mathrm{~K}$ level; and can serve as tracers of large scale convection and interior dynamics. Water and ammonia are condensable species in the Jovian troposphere and of cosmogenic and meteorological importance.

The amount of water in the atmosphere is of particular interest because it indicates Jupiter's oxygen content and the conditions of planet formation and evolution. The Galileo Probe entered a hot spot region and measured the amount of water, finding that the Jovian oxygen content was only $20 \%$ of that expected from solar composition (Niemann et al. 1996). Subsequent measurements of a similar hot spot by NIMS are consistent with the Probe measurements (Carlson et al. 1996).

These results raise the question: Is Jupiter's oxygen content low throughout, the planet having been formed with an oxygen abundance less than solar, or are the low water values simply due to meteorological variations of relative humidity. These two alternative possibilities can be tested by investigating spatial variations in water concentrations.

For many of the Galileo orbits we investigated the water abundance in the 6- to 8-bar level using spectral mapping (Roos-Serote et al. 1997) Two illustrative spectra are shown in Fig. 3, along with line-by-line model fits used to derive water, ammonia, and cloud opacities. The shapes of these two spectra are different, arising from the large differences in water content between the two regions sampled. Again, there is no evidence for a water cloud. A map of the water variations, as well as cloud opacity and relative ammonia abundance, is shown in Fig. 4 (see color plates). Clearly, water is variable in Jupiter's atmosphere, and the low values inferred by the Probe are not necessarily representative of the entire planet. 


\section{Acknowledgements}

Portions of the work described herein were performed at the Jet Propulsion Laboratory, California Institute of Technology, under contract with the National Aeronautics and Space Administration.

\section{References}

Carlson, R.W. et al. (1992) Space Sci. Rev. 60, pp. 456-502.

Carlson, R. et al. (1996) Science, 274, pp. 385-388.

Irwin, P.G.J. et al. (1996) J. Geophys. Res., 101, 26, pp. 137-26,154.

Irwin, P.G.J. et al. (1997) J. Geophys. Res., submitted.

Niemann, H.B. et al. (1996) Science, 272, pp. 846-848.

Roos-Serote, M. et al. (1997) J. Geophys. Res., submitted. 